\title{
Städtische Philosophenkulte in der griechischen Welt zwischen Archaik und Hellenismus - Fakten und Fiktionen
}

Civic cults for philosophers in the Greek world between the Archaic period and the Hellenistic time - facts and fictions

\section{Matthias Haake}

\section{OpenEdition}

\section{Journals}

\section{Edizione digitale}

URL: https://journals.openedition.org/mythos/1901

DOI: 10.4000/mythos. 1901

ISSN: 2037-7746

\section{Editore}

Salvatore Sciascia Editore

\section{Notizia bibliografica digitale}

Matthias Haake, «Städtische Philosophenkulte in der griechischen Welt zwischen Archaik und

Hellenismus - Fakten und Fiktionen », Mythos [En ligne], 14 | 2020, mis en ligne le 31 décembre 2020, consulté le 07 octobre 2021. URL : http://journals.openedition.org/mythos/1901 ; DOI : https:// doi.org/10.4000/mythos.1901

Questo documento è stato generato automaticamente il 7 octobre 2021. 


\section{Städtische Philosophenkulte in der griechischen Welt zwischen Archaik und Hellenismus - Fakten und Fiktionen}

Civic cults for philosophers in the Greek world between the Archaic period and the Hellenistic time - facts and fictions

\section{Matthias Haake}

In ricordo di Federicomaria Muccioli

\section{I.}

«Alle ehren die Weisen` - diese Aussage des Redners Alkidamas aus dem kleinasiatischen Elaia wird von Aristoteles in seiner Rhetorik als Beispiel für eine logische Argumentationsform angeführt ${ }^{1}$. Dass diese Aussage vom historischen Standpunkt aus betrachtet durchaus nicht unzutreffend ist, ist nicht nur im Text der aristotelischen Rhetorik durch das Anführen von Belegen untermauert, sondern auch in der modernen Forschung verschiedentlich hervorgehoben worden ${ }^{2}$. Unter den von Aristoteles angeführten Weisen befindet sich auch ein Philosoph - Anaxagoras ${ }^{3}$. Doch gibt es neben Anaxagoras noch eine Reihe weiterer Philosophen oder der Philosophie nahestehender Personen aus archaischer, klassischer und hellenistischer Zeit, für die antike Zeugnisse kultische Ehren seitens einer politischen Gemeinschaft überliefern beziehungsweise - vorsichtiger gesprochen - nahelegen. Dabei handelt es sich mit Aristoteles, Athenodoros von Tarsos, Eudamos von Megalopolis, Iollas von Sardis und Pythagoras sowohl um bekannte und zentrale Figuren wie auch um wenig bedeutende Figuren in der Geschichte der Philosophie. 
2 Ziel der nachfolgenden Ausführungen ist eine Analyse von städtischen Kulten für Philosophen andhand der sechs genannten Personen, wobei die Frage nach der Historizität der Kulte sowie nach ihren jeweiligen Begründungen im Zentrum des Interesses steht. Die Untersuchung ist dabei strukturell und methodisch den Arbeiten des Verfassers zu öffentlichen Ehrungen für Philosophen in hellenistischer Zeit verpflichtet, die epigraphisch wie literarisch bezeugt sind ${ }^{4}$. Der kurze Beitrag stellt einen ersten Versuch dar, sich einem Phänomen in umfassender Weise zu widmen, dem in der jüngeren Forschung abgesehen von vereinzelten wichtigen Fallstudien keine Aufmerksam gewidmet worden ist ${ }^{5}$.

II.

\section{Anaxagoras}

3 Gleich der erste $\mathrm{zu}$ behandelnde Fall stellt ein Problem dar: Es ist nicht mit letzter Sicherheit zu entscheiden, ob in der aristotelischen Rhetorik davon die Rede ist, dass Anaxagoras in Lampsakos tatsächlich kultische Ehren erhalten hat oder ob er dort nur in Ehren gehalten worden ist ${ }^{6}$. Geht man davon aus, dass in vorliegender Passage davon die Rede ist, dass Anaxagoras kultisch verehrt worden ist, so ergibt sich eine weitere Schwierigkeit - die Frage nach der Historizität dieser kultischen Ehren. Als gesichert kann nämlich allein angesehen werden, dass sich Anaxagoras in Hellenismus und Kaiserzeit in seinem Geburtsort Klazomenai eines hohen Ansehens erfreute, was seinen Ausdruck in einer Reihe von hellenistischen und kaiserzeitlichen Münzprägungen gefunden hat? ${ }^{7}$.

4 Wie es hingegen um seine Wertschätzung in Lampsakos stand, ist auf Grund der Quellenlage keineswegs sicher. Zwar überliefern neben Aristoteles auch Plutarch und Diogenes Laertios, dass Anaxagoras in Lampsakos hohes Ansehen genossen hätte und letzterer berichtet zudem, dass Anaxagoras dort ehrenvoll bestattet worden $\mathrm{sei}^{8}$, doch gibt es nur ein selten in diesem Zusammenhang diskutiertes Zeugnis ${ }^{9}$, das explizit kultische Ehren für den vorsokratischen Philosophen in seinem selbstgewählten Exil erwähnt: Ailian führt in seinen Vermischten Forschungen aus, dass in Lampsakos ein Altar für Anaxagoras errichtet worden sei, beschrieben auf einer Seite mit den Worten 〈Für den Geist) und auf der anderen mit den Worten «Für die Wahrheit ${ }^{10}$. Allerdings erlaubt diese Textpassage keineswegs einen Rückschluss auf einen Kult für Anaxagoras in Lampsakos, spiegelt sie doch allein den literarischen Umgang mit diesem Thema wider, wie der Altar mit den vorgeblichen Altarinschriften - zweifelsfrei Erfindungen eines unbekannten Autors - in aller Deutlichkeit illustriert. Was die besagten Altarinschriften auf der inhaltlichen Ebene interessant macht, ist die Tatsache, dass sie nahelegen, die Ehren für Anaxagoras mit dessen Philosophie in Verbindung zu setzen etwas, das sich im weiteren Verlauf der Untersuchung als exzeptionelle Begründung für kultische Ehren für einen Philosophen herausstellen wird.

5 Auch wenn nicht mit Sicherheit ausgeschlossen werden kann, dass Anaxagoras in Lampsakos kultische Ehren erhalten hat, so ist es doch zumindest ebenso plausibel, ja eigentlich weitaus wahrscheinlicher, dass es sich bei diesen um eine (im Verlauf des vierten vorchristlichen Jahrhunderts oder noch später entstandene) apologetische biographische Fiktion in Reaktion auf den in der literarischen Tradition bezeugten, in seiner Historizität gleichfalls nicht über jeden Zweifel erhabenen athenischen 
Asebieprozess gegen Anaxagoras handelt, der oftmals als Ursache für Anaxagoras' Ortswechsel von seiner langjährigen Wahlheimat Athen in das kleinasiatische Lampsakos gilt ${ }^{11}$.

\section{Aristoteles}

Und als er (i.e. Aristoteles) in Chalkis starb, schickten sie (i.e. die Stagiriten) nach seinem Körper und errichteten einen Altar an seinem Grab und nannten den Ort Aristoteleion und versammelten dort den Rat ${ }^{12}$.

6 Ist die Vorstellung, dass Aristoteles in seiner Heimatstadt Stageira bestattet wurde in der biographischen Tradition zu Aristoteles durchaus verbreitet ${ }^{13}$, so ist in der antiken Literatur allein in der neoplatonischen Vita Marciana ein Kult für den Wohltäter seiner patris bezeugt. Auch wenn unlängst das Grab des Aristoteles samt Altar in Stageira archäologisch nachgewiesen worden sein soll ${ }^{14}$ : Die Tradition über eine Translation der Gebeine oder der Asche des Aristoteles von seinem Sterbeort Chalkis nach Stageira ist in der biographischen Fiktion aus hellenistischer Zeit zu diesem Philosophen $\mathrm{zu}$ verorten, deren auctor allerdings nicht mehr auszumachen ist - und das Gleiche gilt für Aristoteles' Kult als heros ktistes ${ }^{15}$ : Beides diente Aristoteles' Exkulpierung angesichts der schwerwiegenden, gleichermaßen fiktiven Vorwürfe, die gegen ihn in frühhellenistischer Zeit vorgebracht wurden - darunter insbesondere derjenige, seine Heimatstadt an Philipp II. verraten zu haben ${ }^{16}$.

7 Auch wenn ein Kult für Aristoteles in Stageira in den Bereich der biographischen Fiktion zu verweisen ist, so ist das Beispiel des Aristoteles dennoch in zweifacher Hinsicht instruktiv: Zum einen verdeutlicht es, dass es im Kontext der Ausgestaltung der literarischen Tradition grundsätzlich vorstellbar war, dass eine Person, die Philosoph war, in einer polis kultische Ehren erhielt; zum anderen verweist es auf einen Umstand, der tatsächlich für die wenigen historisch bezeugten Kulte für Philosophen und für mit der Philosophie verbundene Personen in poleis charakteristisch ist: Die kultische Verehrung erfolgte auf Grund politischer Verdienste, nicht aber wegen Leistungen im Feld der Philosophie.

\section{Eudamos von Megalopolis}

8 In aller Deutlichkeit zeigt sich dies auch in den seit nunmehr gut zwei Jahrzehnten bekannten kultischen Ehren für Eudamos in Megalopolis auf der Peloponnes, die durch eine sehr fragmentarische, in das frühe zweite vorchristliche Jahrhundert $\mathrm{zu}$ datierende Inschrift bezeugt sind. Eudamos - so ist ingeniös vorgeschlagen worden sei mit dem literarisch bezeugten temporären Schüler Ekdemos / Ekdelos des akademischen Scholarchen Arkesilaos von Pitane zu identifizieren ${ }^{17}$. Trifft diese keineswegs über jeden Zweifel erhabene Identifizierung das Richtige ${ }^{18}$, dann wäre besagter Eudamos um die Mitte des dritten Jahrhunderts seitens der Megalopoliten mit umfangreichen Ehrungen bedacht worden, wozu auch Opfer für den heros auf seinem Altar gehörten, wobei die Gründe für seine heroischen Ehren in Verdiensten um die Vertreibung des makedonenfreundlichen Tyrannen Aristodamas gelegen hätten ${ }^{19}$ :

Opfern soll die Stadt während des Festes [...] auf dem Altar des Eudamos ein Schaf, so wie es [geopfert wird den anderen] Heroen und den Euergeten des Damos $(. . .)^{20}$. 


\section{Iollas von Sardeis und Athenodoros von Tarsos}

Berücksichtigt man die insgesamt überschaubare Zahl kultischer Ehren, die historischen Personen seitens von Städten verliehen wurden, in Korrelation zum quantitativen Auftreten von Philosophen, so verwundert es nicht, dass kaum Philosophen und mit der Philosophie verbundene Personen kultische Ehren auf Grund eines herausragenden Beitrags zum Wohle der ehrenden politischen Gemeinschaft erhielten, da zwei Aspekte in der zu ehrenden Person zusammenkommen mussten: dass sie Philosoph oder der Philosophie verbunden war und eine kultischer Ehren als würdig erachtete Leistung zum Wohle der ehrenden Gemeinschaft erbracht haben musste.

Zwei Fallbeispiele aus späthellenistischer Zeit, anhand derer sich diese Ausführungen bei aller gebotenen Vorsicht illustrieren lassen, sind Iollas von Sardeis, ein Schüler des akademischen Philosophen Philon von Larissa, und Athenodoros von Tarsos, ein stoischer Philosoph, der dem Umfeld des Augustus zuzurechnen ist und der in seiner Heimatstadt politisch wirkte ${ }^{21}$. Ersterer wurde ob seiner umfangreichen Verdienste als Gesandter, Stratege, Gymnasiarch und Priester der Roma von seiner patris unter anderem mit der Aufstellung dreier agalmata aus Marmor auf umfangreiche Weise geehrt $^{22}$; letzteren ehrten die Tarsier jährlich wie einen Heroen, da er bei Augustus eine Abgabenerleichterung für seine Vaterstadt erlangt hatte ${ }^{23}$ und - wenn man eine zweite Überlieferungslinie ergänzend hinzuzuziehen berechtigt ist - eine Stasiskonstellation in Tarsos nach dem Ende des Bürgerkriegs zwischen Marc Anton und Octavian allem Anschein nach mit Hilfe des Siegers friedlich gelöst haben soll ${ }^{24}$.

\section{Pythagoras}

11 Zum Abschluss gilt es einen ebenso diffizilen wie komplexen Fall anzureißen, der prima facie aus der (spät-)hellenistischen Welt in die Archaik führt, der es aber vielmehr erlaubt - so soll vorgeschlagen werden -, einen zeitlichen Bogen zu den kultischen Ehren für Aristoteles in Stageira und ihrer literarischen Invention zu schlagen. Die Rede ist von Pythagoras, einer der schillerndsten Figuren der griechischen Archaik, die sich eines überaus vielschichtigen Nachlebens bis in die Spätantike und darüber hinaus erfreute - was in ähnlicher Weise auch für die von ihm begründete Gemeinschaft der Pythagoreer zutrifft ${ }^{25}$.

Eine der zahlreichen Facetten der Figur des Pythagoras betrifft ihren kultischen Status. Dabei interessiert hier weder seine Stellung zwischen Menschen und Göttern, übermenschlich und (untergöttlich», die Pythagoras innerhalb der pythagoreischen Gemeinschaft in Kroton zugeschrieben wurde und die Aristoteles in seiner Schrift Über die Pythagoreer skizziert hat ${ }^{26}$, noch geht es um seine göttlichen Qualitäten, die Pythagoras einerseits zu seinen Lebzeiten für sich in Anspruch genommen und in Szene gesetzt haben soll und die ihm andererseits zu Lebzeiten seitens süditalischer städtischer Öffentlichkeiten zugeschrieben worden sein sollen ${ }^{27}$. Vielmehr geht es um die Tradition eines städtischen Kultes, der ihm nach seinem Tod eingerichtet worden sein soll; so heißt es in Iamblichs Schrift Über das Pythagoreische Leben:

Die Metapontier behielten Pythagoras, auch als er nicht mehr unter den Lebenden weilte, im Gedächtnis, weihten sein Haus zum Heiligtum der Demeter und machten aus der Gasse, an der es stand, ein Musenheiligtum ${ }^{28}$. 
keineswegs durchweg positiv konnotiertem Tod, meist im Kontext gewaltsamer innerstädtischer Auseinandersetzungen angesiedelt, und zu seinem Sterbeort ${ }^{31}$, um den es geradezu eine Städtekonkurrenz gegeben zu haben scheint, können an dieser Stelle nicht zum Gegenstand einer eingehenden Analyse gemacht werden. Gleiches gilt auch für die divergierenden Quellenzeugnisse zur lokalen Verortung und der Ausgestaltung von Pythagoras' kultischer Verehrung ${ }^{32}$. Wichtiger ist es in vorliegendem Kontext, die Beobachtung zu deuten, dass für Pythagoras erst - wie oben ausgeführt - seit frühhellenistischer Zeit öffentliche Kultpraktiken bezeugt sind. In Kombination mit den bisherigen Ausführungen zu städtischen Philosophenkulten muss es daher als plausible Hypothese erscheinen, dass es sich bei den literarisch bezeugten kultischen Handlungen für einen um die politische Gemeinschaft verdienten, Rat gebenden Pythagoras um eine spätklassische oder frühhellenistische literarische Invention handelt ${ }^{33}$, die erfolgte, als die kultische Verehrung historischer Personen mit Verdiensten um eine politische Gemeinschaft verstärkt aufzutreten begann ${ }^{34}$, nicht aber um Zeugnisse für eine in der spätarchaischen Zeit etablierte Kultpraxis, die man sich im Gefolge der starken antipythagoreischen Stimmungslage in Süditalien zum Zeitpunkt von Pythagoras' Tod auch nur schwerlich vorstellen mag.

15 Akzeptiert man diese Hypothese, so wäre die Kultfiktion - vergleichbar dem Fall des Aristoteles - inhaltlich in den Diskussionen um die gerade auch in politicis durchaus kontroverse Person des Pythagoras zu verorten ${ }^{35}$, die seit dem vierten vorchristlichen Jahrhundert verstärkt positiv ausgestaltet worden ist, was etwa bei Dikaiarch und Aristoxenos greifbar ist ${ }^{36}$. Die unterschiedlichen, einem Pool an Pythagoras gegenüber positiv eingestellten Traditionen ließen sich dann mit einer Zurückführung auf verschiedene Interessenten an einem heros Pythagoras erklären. Diese wären einerseits in pythagoreischen Milieus, andererseits unter den Produzenten im Feld der Philosophiegeschichtsschreibung und der philosophischen Biographie seit frühhellenistischer Zeit und womöglich in lokalen, krotoniatischen wie metapontischen Kontexten zu suchen, wobei auch die sich seit (spät-)hellenistischer Zeit ausbildenden neopythagoreischen Zirkel keineswegs auszuschließen wären.

\section{III.}

Grundsätzlich ist zu konstatieren, dass städtische Kulte für Philosophen und mit der Philosophie verbundene Personen ein äußerst seltenes Phänomen darstellen, das gesichert nur für die hellenistische Zeit $\mathrm{zu}$ beobachten ist, was sich mit der grundsätzlich $\mathrm{zu}$ beobachtenden zeitlichen Streuung von kultischen Ehren für Sterbliche in nicht-monarchischer Position in Einklang bringen lässt ${ }^{37}$. Grund für diesen Befund ist nicht die Tatsache, dass in der Figur des Philosophen strukturell Aspekte angelegt waren, die grundsätzlich gegen eine kultische Verehrung seitens einer politischen Gemeinschaft gesprochen hätten. Vielmehr - und das macht der 
Begründungsmodus für die kultischen Ehren für Anaxagoras besonders - ist die Ursache in dem Umstand zu suchen, dass die Zuerkennung kultischer Ehren herausragende Verdienste um eine politische Gemeinschaft zur Voraussetzung hatte, worunter Leistungen auf dem Gebiet der Philosophie nicht fielen ${ }^{38}$. Dies zeigt - bei aller gesellschaftlichen Wertschätzung -, dass die Philosophie im öffentlichen polis-Diskurs in Bezug auf die Gemeinschaft als etwas Anderes wahrgenommen wurde als beispielsweise die Dichtung oder der Athletismus, zwei Betätigungsfelder, auf denen es möglich war, ehrenwerte Leistungen zu erbringen, die als Grundlage für öffentliche Kulte als relevant erachtet wurden ${ }^{39}$.

\section{BIBLIOGRAFIA}

BETZ 1983: H.D. Betz, s.v. «Gottmensch II», in Reallexikon für Antike und Christentum XII, Stuttgart 1983, 234-312.

BICKNELL 1967: P.J. Bicknell, «Coins and the Presocratics I - Anaxagoras», Apeiron 1.2 (1967), 26-28.

BOYANCÉ 1936: P. Boyancé, Le culte des Muses chez les philosophes grecs. Étude d'histoire et de psychologie religieuses, Paris 1936.

CARUSO 2014: A. Caruso, «Mouseia pitagorici in Magna Grecia: questioni topografici e culturali», in R. Spadea (a c. di), Kroton. Studi e ricerche sulla polis achea e il suo territorio, Roma 2014, 529-553.

CLAY 2004: D. Clay, Archilochos Heros. The Cult of Poets in the Greek Polis, Cambridge, MA, London 2004.

CLAY 2010: D. Clay, «The Hero Cult of Greek Philosophers», in M. Gagarin (ed.), The Oxford Encylopedia of Ancient Greece and Rome, III, Oxford 2010, 427.

CORNELLI 2013: G. Cornelli, In Search of Pythagoreanism. Pythagoreanism as an Historiographical Category, Berlin, Boston 2013.

CoRnelli, McKirahan, Macris 2013: G. Cornelli, R. McKirahan, C. Macris (eds.), On Pythagoreanism, Berlin, Boston 2013.

DÜRING 1957: I. Düring, Aristotle in the Ancient Biographical Tradition, Göteborg 1957.

FARNELL 1921: L.R. Farnell, Greek Hero Cults and Ideas of Immortality, Oxford 1921.

FLEISCHER 2017: K. Fleischer, «The Pupils of Philo of Larissa and Philodemus' Stay in Sicily (PHerc. 1021, col. 34, 6-19)», Cronache Ercolanesi 47 (2017), 73-85.

FREITAG 2019: K. Freitag, «Griechische Heiligtümer als Handlungsorte und die Ausbildung von Wissenskulturen im antiken Griechenland», in K. Freitag, M. Haake (Hgg.), Griechische Heiligtümer als Handlungsorte. Zur Multifunktionalität supralokaler Heiligtümer von der frühen Archaik bis in die römische Kaiserzeit, Stuttgart 2019, 87-120.

GIANGIULIO 1989: M. Giangiulio, Ricerche su Crotone arcaica, Pisa 1989. 
GIANGIULIO 1995: M. Giangiulio, «Sapienza pitagorica e religiosità apollinea. Tra cultura della città e orizzonti panellenici», in A.C. Cassio, P. Poccetti (a c. di), Forme di religiosità e tradizioni sapienziali in Magna Grecia (Atti del convegno Napoli 14-15 dicembre 1993), Pisa, Roma 1995, 9-27.

Goulet, IZDEBSKA, MACRIS, ProchenKo 2018: R. Goulet, A. Izdebska, C. Macris, K. Prochenko, s.v. «Pythagore de Samos (P 333)», in R. Goulet (éd.), Dictionnaire des Philosophes Antiques, VII, Paris 2018, 681-884, 1025-1174.

HAAKE 2007: M. Haake, Der Philosoph in der Stadt. Untersuchungen zur öffentlichen Rede über Philosophen und Philosophie in den hellenistischen Poleis, München 2007.

HAAKE 2012: M. Haake, «Der Panaitiosschüler Paramonos aus Tarsos, der kappadokische König Ariarathes VI. und eine rhodische Inschrift. Zu Rhodiaka I 1 = SEG XXXIII 642 und Philod. Stoic. Hist. col. LXXIV, II. 5 s. u. col. LXXVII, II. 1-3», Epigraphica 74 (2012), 43-58.

HAAKE 2013: M. Haake, «Illustrating, Documenting, Making-believe. The Use of psephismata in Hellenistic Biographies of Philosophers», in P. Liddel, P. Low (eds.), Inscriptions and Their Uses in Greek and Latin Literature, Oxford 2013, 79-124.

HAAKE 2016: M. Haake, «Asebie als Argument. Zur religiösen Fundierung politischer Prozesse im klassischen und hellenistischen Griechenland: das Beispiel der athenischen Philosophenprozesse», in D. Bonanno, P. Funke, M. Haake (Hgg.), Rechtliche Verfahren und religiöse Sanktionierung in der griechisch-römischen Antike (Akten einer deutsch-italienischen Tagung, Palermo, 11.-13. Dezember 2014 / Procedimenti giuridici e sanzione religiosa nel mondo greco e romano. Atti di un convegno tedesco-italiano, Palermo, 11-13 dicembre 2014), Stuttgart 2016, 207-222.

HAAKE 2019: M. Haake, «Philosophy and the «Mediterranean Wide Web〉. Connecting Elites and Connections in the Upper-Classes of the Graeco-Roman World between the Late Archaic Period and Late Antiquity», in M. Dana, I. Savalli-Lestrade (éds), La cité interconnectée dans le monde grécoromain (IVe siècle a.C.-IVe siècle p.C.). Transferts et réseaux institutionnels, religieux et culturels aux époques hellénistique et impériale, Bordeaux 2019, 307-325.

HABICHT 1988: C. Habicht, «Der Akademiker Iollas von Sardis», Zeitschrift für Papyrologie und Epigraphik 74 (1988), 215-218.

HARLAND 2019: P.A. Harland, "The Most Sacred Society (thiasos) of the Pythagoreans": Philosophers Forming Associations», Journal of Ancient History 7 (2019), 207-232.

JONES 2010: C.P. Jones, New Heroes in Antiquity. From Achilles to Antinoos, Cambridge, MA, London.

KIMMEL-CLAUZET 2013: F. Kimmel-Clauzet, Morts, tombeaux et cultes des poètes grecs. Étude de la survie des grands poètes des époques archaïque et classique en Grèce ancienne, Bordeaux 2013.

KuHN 2014: A.B. Kuhn, «The chrysophoria in the Cities of Greece and Asia Minor in the Hellenistic and Roman Periods», Tyche 29 (2014), 51-87.

LUNT 2009: D.J. Lunt, «The Heroic Athlete in Ancient Greece», Journal of Sport History 36 (2009), 375-392.

MANN, BeNTZ 2001: C. Mann, M. Bentz, «Zur Heroisierung von Athleten», in R. von den Hoff, S. Schmitt (Hgg.), Konstruktionen von Wirklichkeit. Bilder im Griechenland des 5. und 4. Jahrhunderts $v$. Chr., Stuttgart 2001, 225-240.

MojsIK 2015: T. Mojsik, «Arist. Rhet. 2.23.1398b and the Cult of Pythagoras», Classica Cracoviensia 18 (2015), 293-310. 
SCHORN 2018: S. Schorn, «Pythagoras in the Historical Tradition: from Herodotus to Diodorus Siculus», in S. Schorn, Studien zur hellenistischen Biographie und Historiographie, Berlin, Boston 2018, 409-429 [zuerst erschienen in C.A. Huffman (ed.), A History of Pythagoreanism, Cambridge 2014, 296-315].

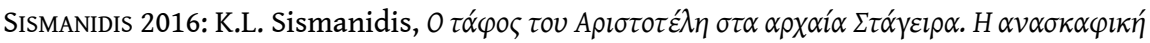
$\alpha \pi \circ \alpha \dot{\lambda} \lambda v \psi \eta$, Thessaloniki 2016.

STAVRIANOPOULOU 2002: E. Stavrianopoulou, «Die Familienexedra von Eudamos und Lydiadas in Megalopolis», Tekmeria 7 (2002), 117-155.

STAVRIANOPOUlou 2017: E. Stavrianopoulou, «Altäre auf den Straßen für die „Söhne des Volkes“», in H. Beck, B. Eckhardt, C. Michels, S. Richter (Hgg.), Von Magna Graecia nach Asia Minor. Festschrift für Linda-Marie Günther zum 65. Geburtstag, Wiesbaden 2017, 281-297.

WALBANK 1957: F.W. Walbank, A Historical Commentary on Polybius. Vol. I: Commentary on Books I-VI, Oxford 1957.

WeLles 1962: C.B. Welles, «Hellenistic Tarsus», in Mélanges offerts au Père René Mouterde pour son 80e anniversaire, 2 vols., Beyrouth 1962, II, 43-75.

ZHMUD 2012: L. Zhmud, Pythagoras and the Early Pythagoreans (original edition Moskau 1994), Engl. transl., Oxford 2012.

\section{NOTE}

1. Die hier vorgelegten Überlegungen stellen die überarbeitete deutsche Version meines in Montpellier gehaltenen Vortrags dar. Flore Kimmel-Clauzet (Montpellier) und Federicomaria Muccioli (Bologna), den spiritus rectores des Projekts Devenir un dieu, devenir un héros en Grèce ancienne / Diventare un eroe, diventare un dio nel mondo greco, gilt mein herzlicher Dank für die Einladung zur Teilnahme an ihrem Unternehmen; den Teilnehmerinnen und Teilnehmern der Tagung weiß ich mich für hilfreiche Anregungen ebenso zu Dank verpflichtet wie den beiden anonymen Gutachtern. Der nachfolgende Text stellt einen ersten Schritt zu einer umfassenden Untersuchung zum Phänomen Philosophenkulte in der Antike dar. - Alkidamas fr. 5 Sauppe = fr. 10 Avezzú = fr. 3 Muir ap. Aristot. Rh. 1398b10-17, hier b10-11.

2. Vgl. etwa JoNES 2010, 42-47; FREITAG 2019, 105.

3. Aristot. Rh. 1398b16. Die Erwähnung des Pythagoras (Aristot. Rh. 1398b15) ist (sehr wahrscheinlich) ein späterer Texteinschub; s. dazu zuletzt MојsIK 2015.

4. Vgl. bes. HAAKE 2007; HAAKE 2013.

5. Hervorgehoben sei an dieser Stelle die - bei aller dem Werk innewohnenden Problematik überaus verdienstvolle, jedoch keineswegs mehr den aktuellen Forschungsstand repräsentierende Arbeit von BOYANCÉ 1936, bes. 229-347. F ARNELL 1921, 361-367 bietet eine Zusammenstellung von einigen Philosophenkulten. Ein kurzer Lexikoneintrag zum Thema stammt von CLAY 2010. B ETZ 1983, 255-286 bietet einen Überblick zu den Aussagen von Philosophen zum Phänomen des «Gottmenschentum».

6. Aristot. Rh. 1398b16. Vgl. allerdings ZHMUD 2012, 144 Anm. 26, der die Ansicht vertritt, dass in vorliegendem Kontext davon ausgzugehen ist, dass Anaxagoras von den Lampsakenern nicht bloß (in Ehren gehalten) wurde, sondern dass der Autor des Texts aussage, dass Anaxagoras tatsächlich kultische Ehren erhalten habe.

7. Vgl. BiCKNELL 1967. 
8. Plut. Mor. 820D; Diog. Laert. II 14-15. Der Text der angeblichen metrischen Grabinschrift findet sich außer in Diog. Laert. II 15 auch in Ail. VH VIII 19 und Anth. Pal. VII 94.

9. Erwähnt von CLAY 2004, 75 mit 173 Anm. 108.

10. Ail. VH VIII 19. Der griechische Text dieses Passus ist sehr unsicher.

11. Zum Asebieprozess gegen Anaxagoras und der Frage seiner Historizität s. H AAKE 2016, 211-212.

12. Vita Aristotelis Marciana 87-90 Gigon (= Aristot. III, p. 29,47-30,3 Gigon) = frg. 18 Düring.

13. Vgl. DÜRING 1957, 232.

14. Vgl. SISMANIDIS 2016.

15. Vgl. dazu HAAKE 2013, 93.

16. Demochares fr. 2 Marasco $=$ BNJ 75 F $2=$ Aristokles F 2.6 Chiesara ap. Euseb. Praep. evang. XV 2, 6. Vgl. dazu HAAKE 2007, 37-38.

17. Vgl. STAVRiANOPOULOU 2002, 131-143, 149-143. Zum Text der Inschrift s. STAVRIANOPOULOU 2002, 120-122; vgl. SEG LII 447-449; BullÉpigr 2005, Nr. 228.

18. Vgl. zurückhaltend HAAKE 2007, 302-304.

19. Vgl. STAVRianopoulou 2002, 138-143.

20. Vgl. StAVRianopoulou 2002, 120 A, Z. 13-15 (SEG LII 447, Z. 13-15) (Übers.: E. Stavrianopoulou). 21. Vgl. STAVRianopoulou 2017, 281 Anm. 2.

22. НАВICHT 1988 hat den in einer späthellenistischen Ehreninschrift aus Sardeis geehrten Iollas, Sohn des Iollas (Sardis VII 1, 27), mit einem in Philodems Index Academicorum bezeugten Philosophenschüler identifiziert (PHerc. 1021, col. XXXIV, 7-8 Dorandi; zu einer verbesserten Lesung von FLEISCHER 2017, 74 und ihrer Bedeutung s. HAAKE 2019, 314 Anm. 47). Entscheidend für die Annahme, dass Iollas in Sardeis kultische Ehren erhielt, ist die Auffassung, dass agalmata in der Bedeutung (Kultbilder) aufzufassen ist; s. dazu HAAKE 2007, 214 Anm. 5. Zu Iollas s. HAAKE 2007, 213-215.

23. [Lucian.] Macr. 21.

24. Str. XIV 5, 14 (674C, 9-675C, 6) Radt. Zu Athenodoros und der Situation in Tarsos s. etwa WelLes 1962, 53-57; HAAKE 2012, 50-51 Anm. 29.

25. Aus der kaum mehr überschaubaren, teilweise sehr konträren Literatur sei exemplarisch verwiesen auf ZHMUD 2012; CORNELLI 2013; CORNELLI, MCKIRAHAN, MACRIS 2013; SCHORN 2018; HARLAND 2019.

26. Aristot. fr. 192 Rose $^{3}=$ fr. 156 Gigon $=$ Pythagoras test. 38 Giangiulio = P 36 Laks $/$ Most ap. Iambl. VP VI 31 [18].

27. Vgl. zum ersten Aspekt etwa Herakleides Pontikos fr. 86 Schütrumpf $=$ fr. $89 \mathrm{Wehrli}^{2}=$ Pythagoras test. 44 Giangiulio = P 37 Laks/Most ap. Diog. Laert. VIII 4-5; Pythagoras P 38 Laks/ Most ap. Porph. VP 45 des Places. Hinsichtlich des zweiten Aspekts, zu dem auf GIANGIULIO 1995, bes.13-27 verwiesen sei, s. beispielsweise Aristot. fr. 191B Rose ${ }^{3}=$ fr. 173 Gigon = Pythagoras test. $37 \mathrm{~b}$ Giangiulio = P 34 Laks/Most ap. Ail. VH II 26; Hieronymos von Rhodos fr. 50 White $=$ fr. 43 Wehrli $^{2}=$ Pythagoras P 40 Laks/Most ap. Diog. Laert. VIII 21; FGrHist 1026 Hermippus of Smyrna F 24 = fr. 20 Wehrli $^{2}=$ Pythagoras test. 87 Giangiulio = P 41 Laks/Most ap. Diog. Laert. VIII 41; Diod. X fr. 3, 2 Cohen-Skalli; FGrHist 1064 Apollonius of Tyana F 1 = Pythagoras P 8 Laks/Most ap. Porph. VP 2 des Places; Diog. Laert. VIII 14; Pythagoras P 35 Laks/Most ap. Iambl. VP VI 30 [18].

28. Iambl. VP XXX 170 [96] (Übers.: M. v. Albrecht).

29. FGrHist / BNJ 566 Timaios von Tauromenion F $131=$ Pythagoras test. 80 Giangiulio $=$ P 46c Laks/Most ap. Porph. VP 4 des Places.

30. Diod. X fr. 23 Cohen-Skalli; Val. Max. VIII 15 ext. 1 Briscoe; Pythagoras P 46b Laks/Most ap. Iust. XX 4, 18; Favorin. fr. 78 Amato = fr. 73 Barigazzi = Pythagoras P 46a Laks/Most ap. Diog. Laert. VIII 15.

31. Vgl. dazu den konzisen Überblick in GoULET, IZDEBSKA, MACRIS, PROCHENKo 2018, 803-804. 
32. Zu den verschiedenen Traditionen in Bezug auf Pythagoras' Tod und Kult vgl. CARUso 2014, 530-548.

33. Verwiesen sei etwa auf Dikaiarchs Ausführungen zu Pythagoras' vier belehrenden Reden an die Bevölkerung Krotons; s. FGrHist 1400 Dikaiarch F 56 = Dikaiarch fr. 40 Mirhady = fr. 33 Wehrli ${ }^{2}$ = Pythagoras test. 47 Giangiulio = P 25 Laks/Most ap. Porph. VP 18-19 des Places.

34. Dieser Kontext ist als terminus post quem für den wohl nachträglich eingeschobenen Hinweis auf Pythagoras' (kultische) Ehren in Aristot. Rh. 1398 b15 (s. Anm. 3) anzusehen.

35. Man denke exemplarisch etwa an Polybios', womöglich, aber keineswegs sicher auf Timaios basierender Darstellung der gewaltsamen anti-pythagoreischen Aufstände in Süditalien; s. Pol. II 39, 1-3 mit WALBANK 1957, 222-224 ad loc. Vgl. in diesem Zusammenhang GiANGiULIO 1989, 26-50.

36. Zum Rat gebenden Pythagoras, angelegt bereits in Isokrates' Busiris (Isokr. Bus. $29=$ Pythagoras test. 17 Giangiulio = P 24 Laks/Most), s. FGrHist 1400 Dikaiarch F $56=$ Dikaiarch fr. 40 Mirhady $=$ fr. $33 \mathrm{Wehrli}^{2}$ test. 47 Giangiulio = P 25 Laks/Most ap. Porph. VP 18-19 des Places. Als Tyrannenfeind beschreibt ihn Aristoxenos; s. Aristoxenos fr. $16 \mathrm{Wehrli}^{2}=$ Pythagoras P 23 Laks/ Most ap. Porph. VP 9 des Places.

37. Instruktiv ist in diesem Zusammenhang ein Vergleich mit der exzeptionellen Ehrung der chrysophoria in hellenistischer Zeit; s. dazu KuHN 2014, 51-63

38. Vgl. in diesem Zusammenhang auch die Ausführungen von HAAKE 2007, 271-285, zur Ehrungspraxis von Philosophen in den hellenistischen poleis.

39. Zu kultischen Ehren für Dichter und Athleten sei verwiesen auf CLAY 2004 und KIMMEL-CLAUZET 2013, 187-271 einerseits sowie MANN, BENTZ 2001 und LUNT 2009 andererseits; s. auch JONES 2010, 38-45.

\section{RIASSUNTI}

Ziel des Aufsatzes ist eine systematische Analyse des Phänomens städtischer Philosophenkulte in der griechischen Welt, die in den literarischen und epigraphischen Quellen für Philosophen archaischer, klassischer und hellenistischer Zeit bezeugt sind. In den Blick genommen werden dabei sechs Philosophen und Personen, die mit der Philosophie in Verbindung standen: Anaxagoras, Aristoteles, Athenodoros von Tarsos, Eudamos von Megalopolis, Iollas von Sardeis und Pythagoras. Im Zentrum des Interesses stehen die Frage nach der Historizität der Kulte sowie nach ihrer argumentativen Funktion im Rahmen der biographischen Darstellungen der behandelten Personen.

The aim of this paper is to provide a systematical analysis of civic cults for philosophers in the Greek world as attested in literary and epigraphic sources for philosophers in Archaic, Classical, and Hellenistic times. It examines six philosophers and persons who were connected with philosophy: Anaxagoras, Aristotle, Athenodorus of Tarsus, Iollas of Sardis, and Pythagoras. The main focus is on questions of the historicity of the cults as well as on their argumentative setting in the context of biographical accounts of the respective persons. 
INDICE

Keywords : philosophers, cults (civic), biographic tradition, Aristotle, Pythagoras

Schlüsselwörter : Philosophen, Kulte (städtisch), biographische Tradition, Aristoteles, Pythagoras

\section{AUTORE}

\section{MATTHIAS HAAKE}

Seminar für Alte Geschichte

Westfälische Wilhelms-Universität Münster

Domplatz 20-22

D-48143 Münster

haakem(at)uni-muenster.de 